\title{
Teleporting a rotation on remote photons
}

\author{
Guo-Yong Xiang, Jian Li, Guang-Can Guo \\ Key Laboratory of Quantum Information, and Department of Physics, \\ University of Science and Technology of China, Hefei 230026 China
}

December 5, 2018

\begin{abstract}
Quamtum remote rotation allows implement local quantum operation on remote systems with shared entanglement. Here we report an experimental demonstration of remote rotation on single photons using linear optical element. And the local dephase is also teleported during the process. The scheme can be generalized to any controlled rotaion commutes with $\sigma_{z}$.
\end{abstract}

PACS: 03.67.Lx, 03.67.Hk, 03.65.Od, 42.50.-P

The development of quantum information in the last two decades promises the powerful applications in information manipulation[1]. Using quantum operations, some missions, for example large number factoring, can be solved by quantum algorithm effectively, which is impossible for classical computer. Arbitrary rotation gates on single qubit and controlled-NOT(CNOT) gate on two qubits are sufficient and necessary, for universal quantum computation[2]. These two kinds of operations have been realized locally in laboratory on physical systems such as trapped ion, neutral atom, and so on[1]. If the qubits involved in the process are separated in distance(e.g. distributed computation[3][4]), operations must be implemented nonlocally via sharing entanglement, local operation and classical communication, i.e. remote operation[5][6][7][8][9][10].

Suppose only two parties, Alice and Bob, are involved in the process[9]. The former has the operation gate, while the later has the state to be operated on. The trivial realisation of remote operation can be finished by the bi-directional quantum state teleportation. The target state is teleported from Bob to Alice. Then Alice applies the operation on the state and sends the resulting state back to Bob via another state teleportation. The total resources for this trivial protocol are two maximally-entangled states (2 ebits) shared and two bits of classical communication (2 cbits) in each direction. And there's no restriction for either the target state or the operation. If the gate is restricted in the unitary operation set $\mathrm{U}_{\text {com }} \cup \mathrm{U}_{\text {anti }}$, where $\mathrm{U}_{\text {com }}\left(\mathrm{U}_{\text {anti }}\right)$ is the set of single qubit operation commuting (anti-commuting) with $\sigma_{z}$, the resource can be reduced to two ebits shared, two cbits from Bob to Alice and one cbit reversely in the optimal nontrivial scheme. Moreover, if the set is either $\mathrm{U}_{\text {com }}$ or $\mathrm{U}_{\text {anti }}$, the scheme can be simplified by using only one ebit shared and one cbit in each direction[9]. Specially, if the operation at Alice's site is a collective one with an auxiliary qubit, the two-qubit gate can be performed nonlocally[5][10].

Here we present an experimental demonstration of a remote rotation on single photons. Unitary rotation commuting with $\sigma_{z}$ is implemented remotely on 
the polarization qubit that Bob hold, using the entanglement between the polarization qubit of one photon and the path qubit of another one from spontaneous parametric down conversion (SPDC).

Let's consider the remote rotation on single qubit. Alice has an operation gate $U_{c o m}=e^{i \varphi \sigma_{z} / 2}$ to implement and Bob has an arbitrary state $|\psi\rangle_{3}=$ $|\psi(\theta, \phi)\rangle_{3}=\alpha|0\rangle_{3}+\beta|1\rangle_{3}$ to be operated on, where $\alpha=\cos \theta, \beta=e^{i \phi} \sin \theta$ and $\{|0\rangle,|1\rangle\}$ are the basis of $\sigma_{z}$. Geometrically, the operation rotates the state vector in the Bloch sphere through angle $\varphi$ about $z$ axis, $|\psi(\theta, \phi)\rangle_{3} \rightarrow$ $\left|\psi\left(\theta, \phi^{\prime}=\phi+\varphi\right)\right\rangle_{3}$ (see Fig.1a). That is the teleportation of an angle. Specially, the gate is an identity operation for $\varphi=0$, while $\sigma_{z}$ for $\varphi=\pi$. Given the shared entanglement $\left|\Phi^{+}\right\rangle_{12}=\frac{1}{\sqrt{2}}\left(|0\rangle_{1}|0\rangle_{2}+|1\rangle_{1}|1\rangle_{2}\right)$, the scheme can be realized by three steps (see Fig.1b).

i) Encoding: Bob performs a CNOT operation on his qubits, 2 and 3, where 3 is the controller. Then a $\sigma_{z}$ measurement on 2 is made by Bob and the result is sent to Alice via one cbit classical communication. Alice would perform bitflip operation $\sigma_{x}$ on her qubit 1 for result $|1\rangle_{2}$, while doing nothing for $|0\rangle_{2}$. The remaining system of qubits 1 and 3 , are in state

$$
|\psi\rangle_{13}=\alpha|0\rangle_{1}|0\rangle_{3}+\beta|1\rangle_{1}|1\rangle_{3} .
$$

Thus the state $|\psi\rangle_{3}$ to be operated on is encoded into $|\psi\rangle_{13}$ within the Hilbert subspace $H^{2}=\left\{|0\rangle_{1}|0\rangle_{3},|1\rangle_{1}|1\rangle_{3}\right\}$ of the composite system, 1 and 3 .

ii) Operating: Alice implements the required quantum gate $U_{\text {com }}$ on her qubit 1, while Bob do nothing,

$$
U_{\text {com }} \otimes I_{3}|\psi\rangle_{13}=\alpha e^{i \varphi / 2}|0\rangle_{1}|0\rangle_{3}+\beta e^{-i \varphi / 2}|1\rangle_{1}|1\rangle_{3} .
$$

Here the local gate plays the role of global rotaion within $H^{2}$. The desired state $\left|\psi^{\prime}\right\rangle_{3}=U_{\text {com }}|\psi\rangle_{3}$ has been just embedded in the composite system.

iii) Decoding: A $\sigma_{x}$ measurement $\left\{| \pm\rangle=\frac{1}{\sqrt{2}}(|0\rangle \pm|1\rangle)\right\}$ is performed on 1 by Alice and the result is sent to Bob. Qubit 3 will be found in the desired state $\left|\psi^{\prime}\right\rangle_{3}$ for the result $|+\rangle_{1}$ or $\left|\psi^{\prime}\right\rangle_{3}=U_{c o m} \sigma_{z}|\psi\rangle_{3}$ in for $|-\rangle_{1}$. The latter can be converted into $\left|\psi^{\prime}\right\rangle_{3}$ by an additional $\sigma_{z}$ rotation for $\sigma_{z} U_{c o m} \sigma_{z}=U_{c o m}$. That is, $\left|\psi^{\prime}\right\rangle_{3}$ is decoded out from the composite system.

The total resources needed in the whole process are one ebit shared, one cbit from Bob to Alice for encoding and one cbit from Alice to Bob for decoding.

To realise the protocol above, the key is how to choose physical qubit, local operation gate and entangled state. For photons, both the polarization $\{|H\rangle,|V\rangle\}$ and the path $\{|u\rangle,|d\rangle\}$ can represent the logic states $\{|0\rangle,|1\rangle\}$ for qubits. For polarization qubit, arbitrary unitary rotation can be performed by using half-wave plate(HWP) and quarter-wave plate(QWP)[11]. The controlledNOT gate between polarization qubit and path qubit of the same photon can be operated on by the polarization beam splitter(PBS), $|H\rangle|u\rangle(|H\rangle|d\rangle) \rightarrow$ $|H\rangle\left|u^{\prime}\right\rangle\left(|H\rangle\left|d^{\prime}\right\rangle\right),|V\rangle|u\rangle(|V\rangle|d\rangle) \rightarrow|H\rangle\left|d^{\prime}\right\rangle\left(|H\rangle\left|u^{\prime}\right\rangle\right)[12]$. And the bi-photon states entangled between polarization or path qubit can be generated via SPDC process[13]. Here the three qubits are embedded in the three freedoms of two photons distributed to Alice and Bob, polarization(qubit 1) of photon $A$, path(qubit 2) and polarization(qubit 3) of photon $B$.

The experimental setup is shown in Fig.2. A mode-locked Ti:Sapphire pulsed laser (with the pulse width less than $200 \mathrm{fs}$, the repetition about $82 \mathrm{MHz}$ and the 
center-wavelength at $780.0 \mathrm{~nm}$ ) is frequency-doubled to produce the pumping source for SPDC process. A $1.0 \mathrm{~mm}$ thick BBO crystal cut for type-II phase match is used as the down converter. By the non-collinear degenerated SPDC procss, two photons, $A$ and $B$, are produced in the polarization-entangled state $\left|\Psi^{+}\right\rangle_{A B}=\frac{1}{\sqrt{2}}\left(|H\rangle_{A}|V\rangle_{B} \pm|V\rangle_{A}|H\rangle_{B}\right)[13]$. Bob uses PBS P1 to split photon $B$ in two paths $\{|u\rangle,|d\rangle\}$ and HWP $H 1$ at $45^{\circ}$ as a $\sigma_{x}$ gate is used to flip the polarization in path $u$. Hence the polarization entanglement between the two photons is converted into polarization-path entanglement,

$$
\left|\Psi^{+}\right\rangle_{123}=\frac{1}{\sqrt{2}}\left(|H\rangle_{1}|u\rangle_{2}+|V\rangle_{1}|d\rangle_{2}\right)|H\rangle_{3} .
$$

The polarization of $B$ can be prepared in arbitrary state $|\psi\rangle_{3}=\alpha|H\rangle_{3}+\beta|V\rangle_{3}$ with identical sets of waveplates, $\left\{H_{u}, Q_{u}\right\}$ and $\left\{H_{d}, Q_{d}\right\}$, in each path[11]. The global state is initialized in $\left|\Phi^{+}\right\rangle_{12}|\psi\rangle_{3}=\frac{1}{\sqrt{2}}\left(|H\rangle_{1}|u\rangle_{2}+|V\rangle_{1}|d\rangle_{2}\right)\left(\alpha|H\rangle_{3}+\right.$ $\left.\beta|V\rangle_{3}\right)$. The three steps for remote rotation are performed as follows.

$\left.i^{\prime}\right)$ Encoding: Path $u$ and $d$ of photon $B$ are input in a PBS P2 to perform a CNOT operation, where polarization is the controlling qubit and path is the target one. The optical path length of $u$ and $p$ are tuned to be equal to ensure no relative phase factor between the two terms in eq.3. The $\sigma_{z}$ measurement on qubit 2 is completed by reading out the path information of photon $B$. If $B$ is found in path $u^{\prime},|\psi\rangle_{B}$ is encoded into $|\psi\rangle_{A B}=\alpha|H\rangle_{A}|H\rangle_{B}+\beta|V\rangle_{A}|V\rangle_{B}$. Or if $B$ is found in path $d^{\prime}$, the two photons will be in $\left|\psi^{\prime}\right\rangle_{13}=\alpha|V\rangle_{1}|H\rangle_{3}+$ $\beta|H\rangle_{1}|V\rangle_{3}$, which can be transformed into $|\Psi\rangle_{13}$ by another HWP at $45^{\circ}$ on photon $A$. Here we omit the later case without loss of generality. The polarization state of photon $B$ is encoded in $\left\{|H\rangle_{1}|H\rangle_{3},|V\rangle_{1}|V\rangle_{3}\right\}[14]$.

ii') Operating: The operation $U_{\text {com }}$ can be performed by a pair of QWP at $45^{\circ}$ with a HWP at $\frac{\varphi}{2}-45^{\circ}$ between them. Such device has been used to verify the geometric phase of classical light and photons[15][16]. For single qubit operation, any additional global phase is trivial, so $U_{c o m}$ can be replaced by $e^{i \varphi / 2} U_{c o m}$, which can be realised by one zero-order waveplate at $0^{\circ}$ tilted in a suitable angle(see ref.[17] for similar application). Here we chose $\varphi=120^{\circ}$ by a tilted QWP Q1.

iii') Decoding: Alice makes her $\sigma_{x}$ measurement $\left\{|D\rangle_{1}=\frac{1}{\sqrt{2}}\left(|H\rangle_{1}+|V\rangle_{1}\right),|C\rangle_{1}=\right.$ $\left.\frac{1}{\sqrt{2}}\left(|H\rangle_{1}-|V\rangle_{1}\right)\right\}$ using polarizer. Photon $A$ is detected by a single photon detector(SPCM-AQR-14 by EG\&G). Photon $B$ will be collapsed into $\left|\psi^{\prime}\right\rangle_{3}=$ $U_{\text {com }}|\psi\rangle_{3}$ for result $|+\rangle_{1}$, and $\left|\psi^{\prime \prime}\right\rangle_{3}=U_{\text {com }} \sigma_{z}|\psi\rangle_{3}$ for result $|-\rangle_{1}$. The latter can be converted into $\left|\psi^{\prime}\right\rangle_{B}$ by a HWP at $0^{\circ}$, i.e. a $\sigma_{z}$ rotation. The ploarization state of photon $B$ is reconstructed by quantum state tomography using polarization analyzer and detector. The measurement on $A$ and $B$ are collected for coincidence count with the window time $5 \mathrm{~ns}$.

In the real experiment, there are two kinds of imperfection which induce the phase decoherence. One is caused by the birefregency of BBO, which induces the partial time-seperation between the wavepackets of two polarizations. It can be described by Kraus operators $\left\{\sqrt{\frac{1+p}{2}} I, \sqrt{\frac{1-p}{2}} \sigma_{z}\right\}$ on photon $A$ or $B$. Here $p$ is just the visibility of the entangled state from SPDC. The other one is the mismatching of space mode in PBS P2(see Fig.3). The two PBSs, P1 amd P2 at Bob's site consist of a Mach-Zedner interferometer. The mode-mismatch can be represented by a process dephase $\left\{\sqrt{\frac{1+\eta}{2}} I, \sqrt{\frac{1-\eta}{2}} \sigma_{z}\right\}$ on the paths of $B$, where 
$\eta$ is the visibility of interferometer. Because the symmetry between two qubit in $\left|\Phi^{+}\right\rangle$, both of the imperfections can be considered to be performed on the polarization of photon $A$. Further, it can also be regarded as a control phase operation on qubit 1 and an anxiliary system 1', where the later is the target. Since the control phase gate $C P_{1^{\prime} 1}=|0\rangle_{1^{\prime} 1^{\prime}}\langle 0|\otimes I+| 1\rangle_{1^{\prime} 1^{\prime}}\langle 1| \otimes \sigma_{z}$ commuting with $\sigma_{z}$ on 1 , the dephasing, or the control phase operation in the extended systems, is included in $\mathrm{U}_{\text {com }}$. That is the nonlocal implement of a control phase gate. With a $\sigma_{x}$ operation on 1', the nonlocal $C N O T$ has been demonstrated recently, where qubit 1 and 1 ' are path and polarization of photon $A[10]$. Moreover, it can be generalized to the controlled rotation $C R=|0\rangle_{1^{\prime} 1^{\prime}}\left\langle 0\left|\otimes U_{\text {com }}+\right| 1\right\rangle_{1^{\prime} 1^{\prime}}\langle 1| \otimes U_{\text {com }}^{\prime}$ for any $U_{c o m}$ and $U_{c o m}^{\prime}$. In our experiment, $1^{\prime}$ is the wavepacket distinction and the mode-mismatch, both of which are traced out. So both the rotation and the dephase on photon $A$ are teleported. The dephased operation is a complete positive map $\varepsilon_{d}=\left\{\sqrt{\frac{1+p \eta}{2}} U_{c o m}, \sqrt{\frac{1-p \eta}{2}} U_{c o m} \sigma_{z}\right\}$. The final state after operation is

$$
\rho_{d}(|\psi\rangle)=\varepsilon_{d}(|\psi\rangle)=\left(\begin{array}{cc}
\alpha \alpha^{*} & p \eta \alpha \beta^{*} e^{-i 2 \varphi} \\
p \eta \alpha^{*} \beta e^{i 2 \varphi} & \beta \beta^{*}
\end{array}\right) .
$$

To completely characterize the remote operation $\varepsilon_{e}$ in our experiment, four state $\left\{|H\rangle,|V\rangle,|D\rangle,|R\rangle=\frac{1}{\sqrt{2}}(|H\rangle-i|V\rangle)\right\}$ are input for the quantum process tomography[1][20]. The process is represented by a positive Hermitian matrix $\chi=\left\{\chi_{m n}\right\}$, which satisfies $\varepsilon(\rho)=\sum_{m n} \chi_{m n} E_{m} \rho E_{n}^{\dagger}\left(\left\{E_{m}\right\}=\left\{I, \sigma_{x}, \sigma_{y}, \sigma_{z}\right\}\right)$. The matrix are shown in Fig.4 for ideal rotation $\chi_{i}$, dephased rotation $\chi_{d}$, and effective operation $\chi_{e}$ in our experiment, where two parameters for $\varepsilon_{d}$ are measured, $p \approx 0.85$ and $\eta \approx 0.92$. And the comparison of experimental operation $\varepsilon_{e}$ with the dephased one $\varepsilon_{d}$ is characterised by the average fidelity of pure state inputted throughout the Bloch sphere $\bar{F}\left[\varepsilon^{\prime}, \varepsilon\right]=\int d \psi F\left[\varepsilon^{\prime}(|\psi\rangle), \varepsilon_{d}(|\psi\rangle)\right]$, where $F\left[\rho, \rho^{\prime}\right]=\operatorname{Tr}\left[\sqrt{\left.\sqrt{\rho^{\prime}} \rho \sqrt{\rho^{\prime}}\right]}[1][18][19]\right.$. From $\chi$ we get $\bar{F}\left[\varepsilon_{i}, \varepsilon_{e}\right]=0.96$ and $\bar{F}\left[\varepsilon_{d}, \varepsilon_{e}\right]=0.99$. Geometrically, the rotation can also be characterized by the actual rotation angle $\varphi=\phi^{\prime}-\phi$. Here we use the angle deviation $\delta=\delta\left(\rho_{i}, \rho_{e}\right)$, which is defined by the cross angle between Bloch vector by ideal rotaion and the one we finally get, to characterize the experimental operation. The maximal angle is $\delta_{\max }=7^{\circ}$, that means $\varphi=120^{\circ} \pm 7^{\circ}$.

In conclusion, a remote rotation on qubits throught $120^{\circ}$ about $z$ axis is performed using shared entanglement and local operation without rotate the target photons. And the dephase on photon $A$ is also be teleported. The whole process is measured by quantum process tomography and agrees with the theoretical prodiction. The scheme can be generalized to remote implement a class of controlled rotation. Although only rotaion commuting with $\sigma_{z}$ is used in our experiment, it's the same with operations anti-commuting with $\sigma_{z}$.[9]

The authors acknowledge Yun-Feng Huang and Xi-Feng Ren for technique help. This work was supported by the Chinese National Fundamental Research Program (2001CB309300), the NSF of China (10304017), the Innovation funds from Chinese Academy of Sciences.

\section{References}

[1] M. A. Nielsen and I. L. Chuang, Quantum Computation and Quantum Information (Cambridge University Press, Cambridge, 2000) 
[2] M. Reck, A. Zeilinger, H. J. Bernstein, and P. Bertani, Phys. Rev. Lett., 73,58 (1994)

[3] L. K. Grover, arXiv e-print:quant-ph/9607024

[4] J. I. Cirac, A. K. Ekert, S. F. Huelga, and C. Macchiavello, Phys. Rev. A, 59, 4249 (1999)

[5] J. Eisert, K. Jacobs, P. Papadopoulos, and M. B. Plenio , Phys. Rev. A, 62, $052317(2000)$

[6] S. F. Huelga, J. A. Vaccaro, A. Chefles and M. B. Plenio, Phys. Rev. A, 63, 042303 (2001).

[7] D. Collins, N. Linden, and S. Popescu, Phys. Rev. A, 64, 032302 (2001)

[8] B. Reznik, Y. Aharonov, and B. Groisman, Phys. Rev. A, 65, 032312 (2002)

[9] S. F. Huelga, M. B. Plenio, and J. A. Vaccaro, Phys. Rev. A, 65, 042316 (2002)

[10] Y. F. Huang, X. F. Ren, Y. S. Zhang, L. M. Duan, and G. C. Guo, accepted by Phys. Rev. Lett., also arXiv e-print:quant-ph/0408007

[11] D. F. V.James, P. G. Kwiat, W. J. Munro, and A. G. White, Phys. Rev. A, 64, $052312(2001)$

[12] N. J. Cerf, C. Adami, and P. G. Kwiat, Phys. Rev. A 57, R1477 (1998)

[13] P. G. Kwiat, K. Mattle, H. Weinfurter, A. Zeilinger, A. V. Sergienko, and Y. Shih, Phys. Rev. Lett., 75, 4337 (1995)

[14] T. B. Pittman, B. C. Jacobs, and J. D. Franson, Phys. Rev. A, 69, 042306 (2004)

[15] P. Hariharan and M. Roy, J. Mod. Opt., 39, 1811 (1992)

[16] J. Brendel, W. Dultz, and W. Martienssen, Phys. Rev. A, 52, 2551 (1995)

[17] P. G. Kwiat, E. Waks, A. G. White, I. Appelbaum, and P. H. Eberhard, Phys. Rev. A, 60, R773 (1999)

[18] M. D. Bowdrey, et.al., Phys. Lett. A, 294, 258 (2002)

[19] M. A. Nielsen, Phys. Lett. A, 303, 249-252 (2002)

[20] J. B. Altepeter, D. Branning, E. Jeffrey, T. C. Wei, P. G. Kwiat, R. T. Thew, J. L. O'Brien, M. A. Nielsen, and A. G. White, Phys. Rev. Lett., 90, $193601(2003)$

Figure 1. a. Geometrical interpretation of single qubit rotaion $U_{\text {com }}$, $\left|\psi^{\prime}\right\rangle=U_{\text {com }}|\psi\rangle$; b. Quamtum circuit for schem of remote rotaion on single qubit, where qubit 1 and 2 are entangled and qubit 3 is the target to be operated on. The whole process is devided into three steps (see text for details). 
Figure 2. Experimental setup to perform a remote rotation on a single photon. P1, P2: polarization beam splitters; $H_{1}, H_{u}, H_{d}$ :half wave plates; $Q_{1}, Q_{u}, Q_{d}$ : quarter wave plates; P:polarizer; PA:polarization analyzer; IF: interference filter; $D_{1}, D_{2}$ :single photon detectors.

Figure 3. Schematic drawing for the mode-mismatching in polarization beam splitter, which induces a dephase.

Figure 4. $\chi$ matrices determined by a) ideal rotaion $\chi_{i}$, b) dephased rotaion $\chi_{d}$, and c) experimental rotaion $\chi_{e}$ from fig. 2 . The real parts of $\chi$ are on the left while the imaginary ones are on the right. 